\title{
Review of: "FMRP inhibits translation elongation independent of mRNA G-quadruplexes"
}

\author{
Yue-Sheng Long ${ }^{1}$ \\ 1 Guangzhou Medical University
}

Potential competing interests: The author(s) declared that no potential competing interests exist.

It is an interesting work that expands the role of FMRP in the translational regulation in the cells. This paper is well designed and written, and its conclusion is convincing. 